\title{
Idiopathic Eosinophilic Pneumonias
}

\section{Einleitung}

Innerhalb der interstitiellen Lungenerkrankungen stellen die idiopathischen eosinophilen Pneumonien Entitäten dar, deren diagnostisches Hauptmerkmal die Vermehrung eosinophiler Granulozyten ist.

In einer Reihe entzündlicher, parasitärer und auch neoplastischer Prozesse wird jeweils eine Vermehrung dieser Zellelemente beobachtet. Wie die eosinophilen Zellen in den jeweils pathogenetischen Prozess einbezogen sind, ob fördernd oder bremsend, ist bislang weitgehend unbekannt.

Auch der eosinophile Granulozyt (so genannt wegen seiner eosinophil anzufärbenden Granula) ist von den Knochenmarkstammzellen abgeleitet. Er besitzt zwei Arten von Granula. In den größeren Granula ist unter anderem das kationische Protein enthalten. Die schmaleren Granula sind von amorpher Natur und enthalten Sulfatasen und Phosphatasen [11]. Unter dem Einfluss von Interleukin-5 und Wachstumsfaktoren gelangt der Eosinophile in die Blutbahn, um sie 24 Stunden später in Richtung Gewebe wieder zu verlassen. Die eosinophilen Zellen prozessieren inflammatorische Mediatoren [46,60] und enzymatische Proteine und geben sie in das umgebende Gewebe ab [9]. Dabei kommt es unter Einfluss der T-Helferzellen zu Interaktionen mit Mastzellen, Makrophagen, Thrombozyten, Fibroblasten und auch Endothelzellen (Abb.1).

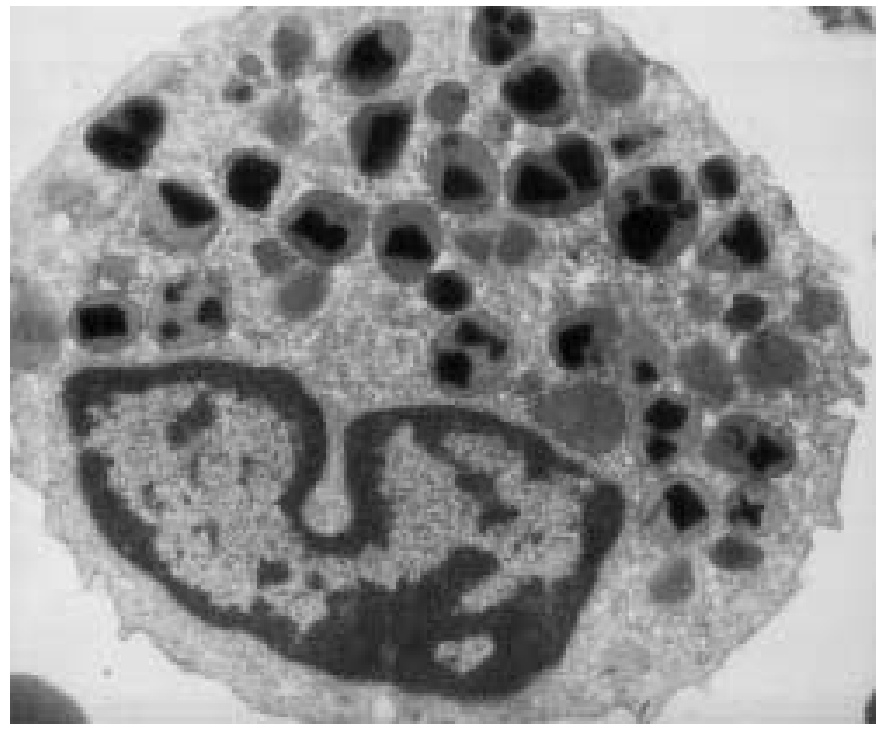

Abb. 1 Elektronenmikroskopische Aufnahme eines eosinophilen Granulozyts von Prof. Dr. K. F. Rabe, Leiden/NL freundlicherweise zur Verfügung gestellt.

\section{Geschichte}

In einer Publikation über Fälle von Lungeninfiltraten mit Eosinophilie berichteten bereits 1952 Reeder u. Goodrich [66] über ätiologisch verschiedenartige Lungenerkrankungen mit dominanter Eosinophilie. Zwei der berichteten acht Fälle sind im Nachhinein vereinbar mit einer chronischen eosinophilen Pneumonie (zitiert nach [18]). 
Tab. 1 Einteilung „pulmonaler Eosinophilien“ nach Crofton u. Mitarb. [20]

Crofton u. Mitarb. [20] berichteten im gleichen Jahr über 450 Fälle (!) von „pulmonaler Eosinophilie“ und steuerten 16 eigene Fälle hier hinzu. Sie klassifizierten ihre Beobachtungen in fünf verschiedene Krankheitsgruppen (Tab.1).

In einer Serie mit 27 Beobachtungen über „cryptogenic pulmonary eosinophilia" von McCarthy u. Pepys vergleichen die Autoren die klinischen Bilder mit Fällen von allergischer bronchopulmonaler Aspergillose (ABPA) [53]. 1969 publizierten Carrington u. Mitarb. die ersten Berichte über Fälle von chronischer eosinophiler Pneumonie [15]. Über genetische Dispositionen gibt es wenige Berichte. Barnes u. Mitarb. berichteten über CEP bei eineiigen Zwillingen [8]. Die erste Publikation über eine akute Verlaufsform dieser Erkrankung verdanken wir Davis im Jahre 1986 [22]. In den letzten Jahren mehren sich auch in Europa die Beobachtungen derartiger meist dramatisch verlaufender Krankheiten $[2,6,17,27]$.

Bisher gibt es keine allgemein akzeptierte Klassifikation der eosinophilen Pneumonien. Für klinisch praktische Belange eignet sich die von Cordier 1998 vorgeschlagene Einteilung [18] (s. Tab. 2). Es wird grundsätzlich zwischen eosinophilen Pneumonien unbekannter Ätiologie und bekannter Ursache unterschieden. Als dritte Kategorie werden die häufigsten Syndrome mit Eosinophilie benannt.

\section{Pathologische Anatomie}

Pathologisch anatomisch unterscheiden sich eosinophile Pneumonien bekannter und unbekannter Ursache nicht. Entscheidend ist der Nachweis bzw. die Präsenz als kausal angesehener Agentien in den Präparaten wie Parasiten oder Pilzhyphen. Bei medikamentöser eosinophiler Pneumonie gibt die Anamnese erste Hinweise auf die Ätiologie. Das entscheidende Merkmal ist die Ausfüllung der Alveolarräume durch eosinophile Zellen. Darüber hinaus sind Makrophagen in den Präparaten ebenfalls vermehrt vorhanden. Einige Riesenzellen enthalten Charcot-Leyden-Kristalle. Eosinophile Mikroabszesse können beobachtet werden.

Elektronenmikroskopische Untersuchungen zeigen, dass sich die eosinophilen Zellen in einem unterschiedlichen Degranulationszustand befinden. Die Makrophagen enthalten unterschiedliche Anteile eosinophiler Granula und Zellfragmente. Immunhistochemische Untersuchungen führen den Nachweis von major basic protein (MBP) und eosinophilem kationischen Protein (ECP) in den eosinophilen Zellen.
Tab. 2 Klassifikation eosinophiler Pneumonie nach Cordier [18]

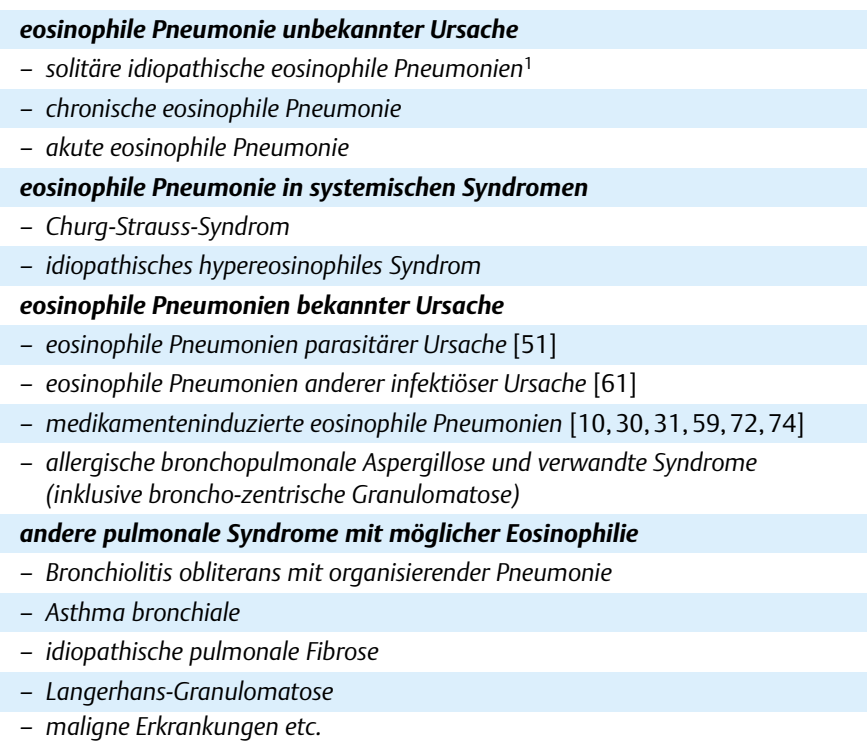

${ }^{1}$ Löffler's Syndrom kann auch idiopathisch sein

Die interstitielle Entzündung beruht also auf der Ansammlung von Eosinophilen, Lymphozyten, Plasmazellen und Histiozyten. Der Fibrosierungsgrad der chronischen eosinophilen Pneumonien ist abhängig von der Dauer der Entzündung und deshalb unterschiedlich ausgeprägt. Die Alveolararchitektur ist in der Regel erhalten. Eine zusätzliche geringgradige Vaskulitis wird gelegentlich beobachtet (Abb. 2).

Die erste Arbeit von Carrington u. Mitarb. weist bereits auf eine Besonderheit hin, die immer wieder bei den Fällen von chronisch eosinophiler Pneumonie beobachtet werden kann: zusätzlich vorhandene Bronchiolitis-obliterans-ähnliche Bilder im Sinne eines BOOP-pattern. Die Verteilung der pneumonischen Infiltrate ist meist beidseitig und diffus, in einigen Fällen jedoch auch einseitig und streng fokal. Hiläre Lymphknotenvergrößerungen sind selten vorhanden.

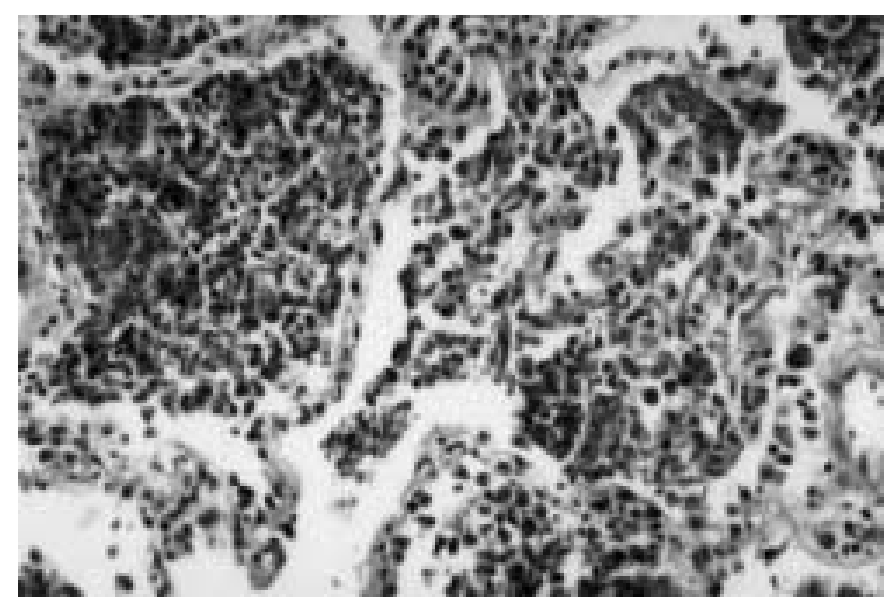

Abb. 2 Lungenbiopsie einer chronisch-eosinophilen Pneumonie; freundlicherweise von Prof. M. Amthor/Rotenburg zur Verfügung gestellt. 
Für detaillierte pathologisch-anatomische Beschreibungen der eosinophilen Pneumonien wird auf die entsprechenden Textbücher verwiesen $[21,28,41]$.

\section{Chronische eosinophile Pneumonie}

\section{Epidemiologie}

Die chronische idiopathische eosinophile Pneumonie (CEP) betrifft vorwiegend Frauen (Geschlechtsverteilung ca. 2:1) [38, 50]. Sie ist eine Erkrankung der Erwachsenen, das mittlere Alter beträgt nach der Literatur 45 Jahre [50].

In der Anamnese der Patienten mit CEP finden sich in nahezu der Hälfte der Patienten Hinweise auf eine Atopie, ca. ein Drittel der Patienten leidet gleichzeitig an einem Asthma bronchiale $[38,50]$. Die meisten der Patienten, über die bislang berichtet wurde, waren Nichtraucher.

Angesichts der Seltenheit der Diagnose überrascht die Verzögerung der endgültigen Diagnose von in der Regel 7,7 Monaten [38] nicht.

\section{Klinik}

Eosinophile Pneumonien ohne erkennbare Ursache können entweder akut oder chronisch auftreten. Darüber hinaus gibt es das gleichzeitige Auftreten von eosinophiler Pneumonie und anderen Erkrankungen, wie z. B. das Churg-Strauss-Syndrom, das Hypereosinophile-Syndrom, Sarkoidose oder Morbus Wegener.

Die chronisch eosinophile Pneumonie ist charakterisiert durch schleichenden Beginn von allgemeinen Symptomen wie Müdigkeit, Abgeschlagenheit, Atemnot, Gewichtsabnahme und zunehmender respiratorischer Symptome, insbesondere aber Husten und Belastungsluftnot. Diese Symptome nehmen innerhalb weniger Wochen einen progressiven Verlauf, wobei die Schwere des Krankheitsbildes abhängig von der Ausprägung der radiologischen Veränderungen ist.

Die Tab. 3 u. 4 zeigen die respiratorischen bzw. systemischen Symptome in ihrer Häufigkeit nach der Literatur $[15,25,35$, $38,50,62]$.

Dabei ist die Angabe von Husten auch bei diesen Erkrankungen das Leitsymptom, verbunden mit Fieber, Dyspnoe und einer drastischen Gewichtsabnahme. Letztere wird bei anderen Pneumonien so ausgeprägt selten beobachtet. Die Angabe von Nachtschweiß lässt auch in Anbetracht der beidseitigen oft ungewöhnlichen radiologischen Veränderungen an eine Mykobakteriose denken. Die Sputumfarbe ist im Gegensatz zu bakteriellen Pneumonien nicht rostbraun, sondern weißlich bis gelblich. Erst der Sputumausstrich zeigt die massenhaft vorhandenen eosinophilen Zellen und gibt einen ersten Hinweis auf die Ätiologie der vorliegenden Pneumonie.

\section{Röntgendiagnostik}

Auf der Thoraxübersichtsaufnahme erkennt man meist beidseitige Infiltrationen. Diese Infiltrationen können sich in Form, Größe und Lage ändern (wandernde Infiltrate). Sie sind meist lappenübergrenzend und oft peripher gelegen. In der Übersichts-
Tab. 3 Respiratorische Symptome bei chronisch-eosinophiler Pneumonie (nach Literaturangaben)

$\begin{array}{ll}\text { - Husten } & 90 \% \\ \text { - Dyspnoe } & 57 \text { bis } 90 \% \\ \text { - erhöhte Sputumproduktion } & 30 \text { bis } 40 \% \\ \text { - Thoraxschmerzen } & 10 \text { bis } 16 \% \\ \text { - Hämoptysen } & <10 \%\end{array}$

Tab. 4 Systemische Symptome bei chronisch-eosinophiler Pneumonie (nach Literaturangaben)

\begin{tabular}{ll}
\hline - Fieber & $70 \%$ \\
- Gewichtsabnahme & 50 bis $75 \%$ \\
- Malaise & $50 \%$ \\
- Müdigkeit & $50 \%$ \\
- Nachtschweiß & $80 \%$ \\
\hline
\end{tabular}

Tab. 5 Korrekte Erstdiagnose verschiedener eosinophiler Lungenerkrankungen; Übereinstimmung zwischen Untersuchern (nach [39])

$\begin{array}{ll}\text { chronisch-eosinophile Pneumonie } & 78 \% \\ \text { akute eosinophile Pneumonie } & 81 \% \\ \text { Churg-Strauss-Syndrom } & 44 \% \\ \text { allergische bronchopulmonale Aspergillose } & 84 \% \\ \text { simple pulmonale Eosinophilie } & 17 \% \\ \text { medikamenteninduzierte Eosinophilie } & 27 \%\end{array}$

aufnahme ergibt sich nicht selten das klassische Bild der „photographic negative or reversal of the shadows usually seen in pulmonary edema“. Es wird in ca. $20 \%$ der Fälle gefunden und bietet so erfahrenen Untersuchern in einem beachtenswerten Prozentsatz bereits erste Hinweise auf die Natur der Erkrankung [5,42].

Das hochauflösende CT zeigt die Veränderungen genauer, auch vermeintlich auf eine Seite beschränkte Veränderungen lassen sich dann doch als beidseitige Veränderungen im HR-CT erkennen [57]. Neben dichten Infiltraten (Konsolidierungen) kommt es auch zu nur lose angeordneten Dichteanhebungen (Milchglasmuster) vorwiegend in den oberen Lungenanteilen. Die Infiltrate sind vorwiegend peripher angeordnet, teilweise zeigen sie auch Konsolidierungen auf Segment- oder Lappenebene, gelegentlich führen sie zu Atelektasen [52]. Unter der Kortisontherapie kommt es relativ rasch zur Rückbildung zunächst der Milchglasphänomene, später auch der Konsolidierungen. Die Tab. 5 zeigt aus einer amerikanisch-japanischen Gemeinschaftsarbeit den Grad der Übereinstimmung zwischen zwei Untersuchern bei eosinophilen Lungenerkrankungen [39].

In seltenen Fällen sind computertomographisch auch Hohlraumbildungen beschrieben worden. In bis zu $10 \%$ wird über schmale Ergussbildungen und mediastinale Lymphknotenvergrößerungen berichtet $[26,38,43,50]$. 
Insgesamt ergibt sich bei pneumologisch erfahrenen Radiologen eine brauchbare Übereinstimmung. Der Wert der CT-Untersuchung bei der Diagnostik ist unbestritten. Radiologisch hinweisend sind progrediente dichte Infiltrate in der Lungenperipherie ohne Segment- und Lappenbegrenzung. Bei Rezidiven treten die Infiltrate in gleicher eigenartiger peripherer Lokalisation auf und beweisen beim Vergleich mit den alten Röntgenaufnahmen das Rezidiv. Die radiologische Differenzialdiagnose umfasst naturgemäß andere bakterielle und auch atypische Pneumonien. Das Nichtansprechen auf entsprechende antibiotische Therapien führt meist zur Durchführung eines Thorax-CT. Dabei ergeben sich besondere Probleme der Abgrenzung zur BOOP. Das Beachten der Begleitbefunde gestattet meist erst die korrekte Diagnose [3]. Flaue und wolkige Dichteanhebungen sprechen eher für eine CEP, während bei der BOOP mehr pleuranahe dreieckige Fleckund Streifenschatten beobachtet werden.

\section{Laborwerte}

Ein wichtiges erstes diagnostisches Kriterium ist die periphere Bluteosinophilie, wenn sie wie in $80 \%$ der Fälle mehr als $6 \%$ beträgt. Der mittlere Prozentsatz der Eosinophilen im Differenzialblutbild wird in einer größeren Untersuchung mit 26\% angegeben [38]. Die Bluteosinophilie kann aber auch fehlen. In Fällen mit peripherer Eosinophilie ist meistens die BAL-Eosinophilie deutlich höher als $30 \%$ [50].

Eine Leukozytose wird in $63 \%$ der berichteten Fälle beschrieben Gelegentlich wurden auch Thrombozytosen beobachtet [13]. Die Blutsenkungsreaktion ist in der Regel deutlich beschleunigt. Gleichsinnig ist das C-reaktive Protein stark erhöht. In ca. 50\% ist auch der Gesamt-IgE-Spiegel erhöht, selten auf Werte über $1000 \mathrm{U} / \mathrm{l}$ als differenzialdiagnostisches Kriterium gegenüber der aktiven allergischen bronchopulmonalen Aspergillose [50], bei der die IgE-Spiegel in der Regel über 1000 U/l liegen. Die Bedeutung gelegentlich nachgewiesener zirkulierender Immunkomplexe bei der CEP ist unbekannt und wird einem Epiphänomen zugeschrieben [24], gleiches gilt für den Nachweis von antineutrophilen zytoplasmatischen Antikörpern (pANCA). Eine Sputumeosinophilie wurde in $63 \%$ der untersuchten Fälle in einer Serie gefunden [35].

\section{Bronchoskopie, bronchoalveoläre Lavage (BAL)}

Der endoskopische Befund ist meist unauffällig. Deshalb ist die Gewinnung von Untersuchungsmaterial aus der Lungenperipherie von entscheidender Bedeutung. Die bronchoalveoläre Lavage wird zunehmend für die nicht-invasive Diagnose der eosinophilen Pneumonien genutzt [63]. Im Normalfall werden in der BAL weniger als $1 \%$ eosinophiler Zellen gefunden. Eine leichte Erhöhung der eosinophilen Zellen in der BAL hat wenig diagnostischen Wert und wird bei verschiedensten anderen Erkrankungen, z. B. auch der idiopathischen Lungenfibrose und Kollagenose sowie bei Asthma bronchiale, gefunden. Eine Eosinophilie von mehr als $20-30 \%$ ist mit der Diagnose einer chronisch eosinophilen Pneumonie gut vereinbar $[1,34,37,55,68]$. So wird derzeit die Untersuchung der BAL als entscheidende Methode zur Diagnose einer eosinophilen Pneumonie anerkannt, wenn andere Erkrankungen, die ebenfalls mit höheren Eosinophilenzahlen in der BAL einhergehen können, ausgeschlossen werden. Dabei kann man neben der Vermehrung der eosinophilen Zellen gelegentlich auch Neutrophile, Mastzellen und Lymphozyten beob- achten [50]. Ein wichtiges charakteristisches Kriterium ist der rasche Abfall der Eosinophilenzahl in der BAL unter Kortikosteroidbehandlung [19,23].

Entscheidend ist, dass die BAL in einem radiologisch erkennbar betroffenen Segment durchgeführt wird. Das Thorax-CT sollte also vor der BAL-Untersuchung angefertigt werden.

\section{Histologie}

Zur Diagnose einer eosinophilen Pneumonie gehört der Nachweis der Infiltration des Lungenparenchyms mit eosinophilen Zellen. So ist nur der Nachweis eosinophiler Zellen in großer Zahl aus radiologisch verdächtigen Kompartimenten mit der Diagnose eosinophile Pneumonie vereinbar. Die früher meist geübte Lungenbiopsie ist lediglich vom pathologisch-anatomischen Standpunkt aus weiterhin der Goldstandard für die Diagnose einer eosinophilen Pneumonie, da die histologische Untersuchung neben dem Nachweis eosinophiler Zellen auch den Nachweis von Vaskulitiden gestattet. Histologische Befunde werden naturgemäß den differenzialdiagnostischen Problemen der verschiedenen eosinophilen Erkrankungen am besten gerecht.

Dabei ist heute die offene Lungenbiopsie durch die videoassistierte Thorakoskopie mit Lungenbiopsie ersetzt. Die videoassistierte Biopsie ist sicherer und wenig aggressiv. Die Nebenwirkungsrate ist extrem niedrig $[15,40,54]$.

Transbronchiale Lungenbiopsien sind nur im positiven Falle diagnostisch verwendbar. In den seltensten Fällen gestatten sie aufgrund des Fehlens von Gefäßen die Differenzialdiagnose zu eosinophilen Vaskulitiden. Neben dem Churg-Strauss-Syndrom ist besonders der M. Wegener differenzialdiagnostisch zu beachten $[47,65]$. Über das gleichzeitige Auftreten von CEP und nicht verkäsenden Epitheloidzellgranulomen wurde berichtet [67].

\section{Lungenfunktion bei CEP}

Die Lungenfunktionsuntersuchungen bei Patienten mit CEP zeigen in der Regel restriktive Störungen, gelegentlich sind auch zusätzliche obstruktive Ventilationsstörungen zu finden, letztere vorwiegend bei Patienten mit gleichzeitig bestehendem Asthma bronchiale $[38,50]$. Die meisten der Patienten sind hypoxämisch, stärkere Hypoxämien unter $75 \mathrm{~mm} \mathrm{Hg}$ sind in ca. 64\% der Patienten gefunden worden [50].

\section{Extrapulmonale Symptome und Befunde}

Extrapulmonale Manifestationen sind bei CEP ungewöhnlich und sollten zu einer Überprüfung der Diagnose führen. In seltenen Fällen wurde über Arthralgien und EKG-Veränderungen und Perikarditis berichtet. Auch sind gelegentlich die Leberenzyme erhöht $[4,7,50]$.

\section{Overlap-Syndrom bei CEP}

In extrem seltenen Fällen wird über so genanntes Overlapping gesprochen, d.h., eine chronisch eosinophile Pneumonie ist mit einer anderen Erkrankung vergesellschaftet, wie z.B. ChurgStrauss [69], Sarkoidose [69,71], M.-avium-Infektion [70]. Möglicherweise ist in einigen Fällen auch eine chronisch eosinophile Pneumonie als Vorläufer für ein Churg-Strauss-Syndrom anzunehmen. Durch die gleichartige Therapie wird wahrscheinlich die Ausprägung des Churg-Strauss-Syndroms verhindert. Disku- 
Tab. 6 Differenzialdiagnose zwischen CEP und BOOP (nach Literaturangaben)

\begin{tabular}{|c|c|c|}
\hline & CEP & BOOP \\
\hline Röntgen & $\begin{array}{l}\text { flaue Infiltrate, bevorzugt } \\
\text { OMF, Luftbronchogramm } \\
\text { selten }\end{array}$ & $\begin{array}{l}\text { dreieckförmiges dichtes } \\
\text { Infiltrat in der Lungen- } \\
\text { peripherie (pleuranah!), } \\
\text { bevorzugt MUF, Luft- } \\
\text { bronchogramm häufig }\end{array}$ \\
\hline$B A L$ & Eosinophilie > 20\% & buntes Zellbild \\
\hline Histologie & $\begin{array}{l}\text { keine Fibrose } \\
\text { Schaumzellen selten } \\
\text { BO selten }\end{array}$ & $\begin{array}{l}\text { fibroblastenreich } \\
\text { Schaumzellen häufig } \\
\text { BO häufig }\end{array}$ \\
\hline
\end{tabular}

tabel erscheint auch die Möglichkeit, dass primär ein mildes Churg-Strauss-Syndrom vorliegt, was als chronisch eosinophile Pneumonie missinterpretiert wird [32].

Differenzialdiagnostisch sind neben den bakteriellen und viralen sowie Pilzpneumonien die antibiotikaresistenten Infiltrate zu bedenken. Schwierigkeiten bereitet gelegentlich die Differenzialdiagnose zur BOOP. Die Tab. 6 soll hierbei eine Hilfe geben, wobei immer alle verfügbaren Befunde beachtet werden müssen. Dazu gehören auch Lymphome [12].

\section{Verlauf und Therapie}

Die chronisch eosinophile Pneumonie führt zu einem schweren Krankheitsbild. Bei fehlender korrekter Diagnose sind vielfache antibiotische erfolglose Vorbehandlungen die Regel. Mittel der Wahl bei der CEP sind Steroide. Spontanremissionen wurden jedoch ebenfalls beobachtet $[7,29,49,50]$. Todesfälle werden in der Literatur selten berichtet. Ein Literaturreview [38] berichtet über nur sieben Todesfälle während der Verlaufsbeobachtung. Charakteristisch für die CEP ist die rasche Besserung nach Steroiden. Die Symptome können innerhalb weniger Tage und Wochen völlig verschwinden. Es gibt keine Therapieempfehlungen für die CEP. Allgemein anerkannt sind initiale Tagesdosen zwischen 20 und $60 \mathrm{mg}$ [56]. In der Regel wird man die Dosierung an die bei der Sarkoidose üblichen Dosierungen von 0,5 bis $1 \mathrm{mg}$ pro Kilogramm Körpergewicht anlehnen. Auch geringere Dosen wurden in der Literatur als effektiv erachtet. Neben dem raschen Verschwinden der klinischen Zeichen verbessern sich auch die radiologischen Veränderungen. In der am besten untersuchten Serie von Marchand u. Mitarb. verschwanden die radiologischen Veränderungen innerhalb einer Woche bei 69\% aller Fälle [50].

Die meisten Patienten müssen über viele Monate behandelt werden. Die Mindestdauer der Therapie beträgt sechs Monate. Andernfalls kommt es zu hohen Relaps-Raten bis zu 60\% [38]. Auch die Behandlung der Rückfälle geschieht erneut mit Kortikosteroiden und führt in der Regel ebenso rasch zum Erfolg [7,50].

Der Langzeitverlauf wird häufig von Asthma bestimmt. Asthma trat bei 54\% der Patienten auf und war oft schwergradig und kortikoidpflichtig [50].

Neben systemischen Steroiden war in Einzelfällen auch die Behandlung mit inhalativen Kortikosteroiden mit Dosen um $1500 \mu \mathrm{g} /$ die effektiv. Gelegentlich wurde die Kombination zwi- schen niedrig dosierter oraler Steroidtherapie und Hochdosis-Inhalationstherapie mit Kortikosteroiden beschrieben $[48,56]$. Nur in einem Fall ist bislang die Entwicklung einer Lungenfibrose berichtet worden [75].

\section{Eigene Erfahrungen bei chronisch-eosinophiler Pneumonie (CEP)}

\section{Klinische Daten}

Wir überblicken derzeit den Krankheitsverlauf von 21 Patienten mit CEP und einem Patienten mit AEP [45]. Bei der Einweisung in unsere Klinik war eine derartige Diagnose in keinem Fall in Erwägung gezogen worden. Die Verdachtsdiagnosen differierten von Tuberkulose über atypische Pneumonie bis zu BOOP oder waren nur Beschreibungen der radiologischen Veränderungen, z. B. beidseitig Infiltrate. Die diagnostische Verzögerung betrug im Mittel 5,6 Monate!

Bei allen Patienten wurde die Diagnose einer CEP bzw. AEP aus der Synopsis von klinischem Bild einer Pneumonie, charakteristischen Lungeninfiltraten und dem Ergebnis der BAL (ausgenommen 2 Patienten mit charakteristischer transbronchialer Biopsie und ein Patient mit offener Lungenbiopsie ohne vorherige BAL) gestellt.

Es handelte sich um 19 Frauen und 2 Männer. 17 Patienten waren Nie-Raucher, 2 Patienten Raucher und 2 Ex-Raucher. Husten und Fieber $(n=2)$ waren die führenden Symptome. Die Temperaturerhöhungen betrugen zwischen $37,5-38,5{ }^{\circ} \mathrm{C}$. Septisches Fieber wurde nur bei dem Patienten mit AEP gemessen. 18 Patienten gaben Atembeschwerden, 10 davon sogar Ruhedyspnoe an. $15 \mathrm{~Pa}-$ tienten berichteten über allgemein ausgeprägtes Krankheitsgefühl (Malaise), ebenso viele über Gewichtsabnahme von mehr als 2 Kilogramm in den letzten 4 Wochen. Nur 5 Patienten klagten über Nachtschweiß. Die Charakteristika der 21 Patienten sind in Tab. 7 dargestellt.

\section{Labordaten}

In aller Regel war die Blutsenkungsgeschwindigkeit in einer Stunde deutlich erhöht, nur bei einem Patienten wurde sie mit $8 \mathrm{~mm} / 1$. Std. bestimmt.

Eine Eosinophilie im peripheren Blut über 3 rel.\% diagnostizierten wir in 15 (!) Fällen, über 20 rel.\% allerdings nur bei 3 Patienten. Die Tab. 8 gibt einen Überblick über die Labordaten einschließlich der Funktionsdaten unserer Patienten.

\section{Radiologische Befunde}

Die radiologischen Bilder zeigten meist beidseitige (die Beidseitigkeit war oft nur im CT erkennbar), teils im Verlauf wechselnde Infiltrationen, die auf die unverzüglich eingeleitete Kortikosteroid-Therapie rasch verschwanden. Die Abb.3-6 zeigen die radiologischen Veränderungen vor und nach der Therapie bei $4 \mathrm{~Pa}-$ tienten.

\section{Lungenfunktion}

Die Lungenfunktion war bei 10 Patienten im Sinne einer restriktiven Störung verändert, bei den anderen normal. Eine respiratorische Partialinsuffizienz bestand bei 13 Patienten (Tab. 8). 
Tab. 721 Patienten mit CEP

\begin{tabular}{|c|c|c|c|c|}
\hline $\begin{array}{l}\text { Nr. des } \\
\text { Pat. }\end{array}$ & Alter & Geschlecht & Symptome & $\begin{array}{l}\text { Symptomdauer } \\
\text { (Wochen) }\end{array}$ \\
\hline 1 & 30 & w & $\mathrm{H}, \mathrm{F}, \mathrm{G}, \mathrm{M}, \mathrm{D}$ & 6 \\
\hline 2 & 63 & w & $\mathrm{F}, \mathrm{G}, \mathrm{M}$ & 20 \\
\hline 3 & 50 & w & $\mathrm{H}, \mathrm{F}, \mathrm{G}, \mathrm{M}, \mathrm{D}$ & 16 \\
\hline 4 & 50 & w & $H, F, M, D$ & 20 \\
\hline 5 & 75 & $\mathrm{~m}$ & $\mathrm{H}, \mathrm{F}, \mathrm{G}, \mathrm{M}, \mathrm{D}$ & 82 \\
\hline 6 & 64 & w & $H, F, M, D$ & 6 \\
\hline 7 & 37 & w & $H, F, M, D$ & 8 \\
\hline 8 & 67 & w & $\mathrm{H}, \mathrm{F}, \mathrm{G}, \mathrm{M}, \mathrm{D}$ & 120 \\
\hline 9 & 25 & w & $H, F, D$ & 28 \\
\hline 10 & 20 & w & $H, F, G, D$ & 4 \\
\hline 11 & 66 & w & $\mathrm{H}, \mathrm{F}, \mathrm{G}, \mathrm{M}, \mathrm{D}$ & 16 \\
\hline 12 & 77 & w & $H, F, G, D$ & 72 \\
\hline 13 & 66 & w & $H, F, G, D$ & 13 \\
\hline 14 & 67 & w & $\mathrm{H}, \mathrm{F}, \mathrm{G}$ & 10 \\
\hline 15 & 50 & w & $\mathrm{H}, \mathrm{F}, \mathrm{G}, \mathrm{M}, \mathrm{D}$ & 9 \\
\hline 16 & 62 & w & $\mathrm{H}, \mathrm{F}, \mathrm{G}, \mathrm{M}, \mathrm{D}$ & 7 \\
\hline 17 & 73 & w & $\mathrm{H}, \mathrm{F}, \mathrm{G}$ & 6 \\
\hline 18 & 47 & $\mathrm{~m}$ & $H, M, D$ & 24 \\
\hline 19 & 59 & w & $H, F, M, D$ & 12 \\
\hline 20 & 48 & w & $\mathrm{H}, \mathrm{F}, \mathrm{G}, \mathrm{M}, \mathrm{D}$ & 16 \\
\hline 21 & 59 & w & $\mathrm{H}, \mathrm{F}, \mathrm{G}, \mathrm{M}, \mathrm{D}$ & 4 \\
\hline
\end{tabular}

$\mathrm{H}=$ Husten, $\mathrm{F}$ = Fieber, $\mathrm{G}$ = Gewichtsabnahme, $\mathrm{M}$ = Malaise, $\mathrm{D}=$ Dyspnoe

\section{Endoskopische Befunde}

Die Bronchoskopie erbrachte ein leicht entzündliches Aussehen der Bronchialschleimhaut und wenig Sekretion. Mikrobiologische Untersuchungen blieben steril.

In der BAL, meist aus einem befallenen Segment oder dem Mittellappen, zeigte sich die Kompartimentierung der eosinophilen Entzündung in der Lunge (Tab. 8).

\section{Therapie}

Die Behandlung wurde immer mit 0,3-0,5 mg Prednison/kg KG täglich begonnen. Die Dosis wurde nach klinischer und radiologischer Besserung, frühestens aber nach 4 Wochen auf die Hälfte reduziert und in einer Dosis von $5-15 \mathrm{mg} / \mathrm{d}$ je nach klinischem und radiologischem Bild für weitere 6-9 Monate gegeben. Rezidive wurden bei unserem Klientel 4-mal bei zu früher Dosisreduktion beobachtet. Die erneute Therapie erfolgte nach den gleichen Gesichtspunkten.

\section{Zusammenfassung zur CEP}

Die Diagnose einer CEP wird aufgrund klinischer, radiologischer und gegenwärtig meistens der BAL-Befunde erhoben. In differenzialdiagnostisch schwierigen Fällen wird auch heute eine Lungenbiopsie nicht zu vermeiden sein. Ein wichtiger auch differenzialdiagnostischer Punkt ist das rasche Ansprechen auf Kortikosteroide. Meistens gelingt es innerhalb von wenigen Tagen, das klinische Bild entscheidend zu bessern. Rezidive sind häufig,
Tab. 8 Labor- und Funktionswerte

\begin{tabular}{|c|c|c|c|c|c|}
\hline $\begin{array}{l}\text { Nr. des } \\
\text { Pat. }\end{array}$ & $\begin{array}{l}\text { BSG } \\
(\mathrm{mm})\end{array}$ & $\begin{array}{l}\text { Eos i. Blut } \\
\text { (\%) }\end{array}$ & $\begin{array}{l}\text { Eos i. BAL } \\
\text { (\%) }\end{array}$ & $\begin{array}{l}\text { VC \% } \\
\text { pred }\end{array}$ & $\begin{array}{l}\mathrm{PaO}_{2} \\
(\mathrm{~mm} \mathrm{Hg})\end{array}$ \\
\hline 1 & 79 & 6 & 55 & 43 & 63 \\
\hline 2 & 67 & 3 & 24 & 72 & 73 \\
\hline 3 & 95 & 2 & 18 & 92 & 72 \\
\hline 4 & 36 & 4 & 20 & 113 & 92 \\
\hline 5 & 51 & 3 & 18 & 107 & 60 \\
\hline 6 & 100 & 43 & 90 & 79 & 64 \\
\hline 7 & 42 & 31 & 68 & 94 & 83 \\
\hline 8 & 54 & 6 & 28 & 62 & 43 \\
\hline 9 & 28 & 5 & 17 & 42 & 75 \\
\hline 10 & 90 & 11 & 27 & 59 & 47 \\
\hline 11 & 60 & 32 & 46 & 97 & 94 \\
\hline 12 & 31 & 11 & 49 & 78 & 66 \\
\hline 13 & 51 & 6 & 29 & 122 & 68 \\
\hline 14 & 43 & 12 & 69 & 116 & 73 \\
\hline 15 & 109 & 9 & 37 & 49 & 65 \\
\hline 16 & 113 & 16 & 88 & 69 & 64 \\
\hline 17 & 61 & 15 & 23 & 68 & 78 \\
\hline 18 & 8 & 3 & 31 & 57 & 82 \\
\hline 19 & 93 & 2 & 29 & 92 & 69 \\
\hline 20 & 18 & 14 & 42 & 80 & 73 \\
\hline 21 & 46 & 7 & 39 & 52 & 60 \\
\hline
\end{tabular}

wenn die Mindesttherapiedauer von 6 Monaten nicht eingehalten wird, oder bei zu früher Dosisreduktion.

\section{Akute eosinophile Pneumonie}

\section{Geschichte}

Im Jahre 1986 berichteten Davis u. Mitarb. über die ersten beiden Beobachtungen einer akuten eosinophilen Pneumonie. Die Krankheitsbilder waren derart ausgeprägt, dass eine Beatmungstherapie notwendig wurde. Erstaunlicherweise erholten sich die Patienten nach einer Kortikosteroidtherapie rasch und andauernd. In der Folge sind zunächst nur in Amerika [2] und später auch in anderen Ländern derartige Krankheiten diagnostiziert und als Fallberichte publiziert worden $[2,6,14,16,17,27,33,36$, $44,58,64]$.

\section{Epidemiologie}

Aus der Literatur ist bekannt, dass es sich bei den Patienten vorwiegend um jüngere Patienten (im Mittel 30 Jahre alt) handelt $[6,17,29]$, einige Patienten haben eine Atopie-Anamnese. Die vorwiegende Erkrankungszeit im Frühling und Sommer lässt Spekulationen zu Umweltallergenen zu. Diese wurden jedoch bisher nicht eindeutig identifiziert. 

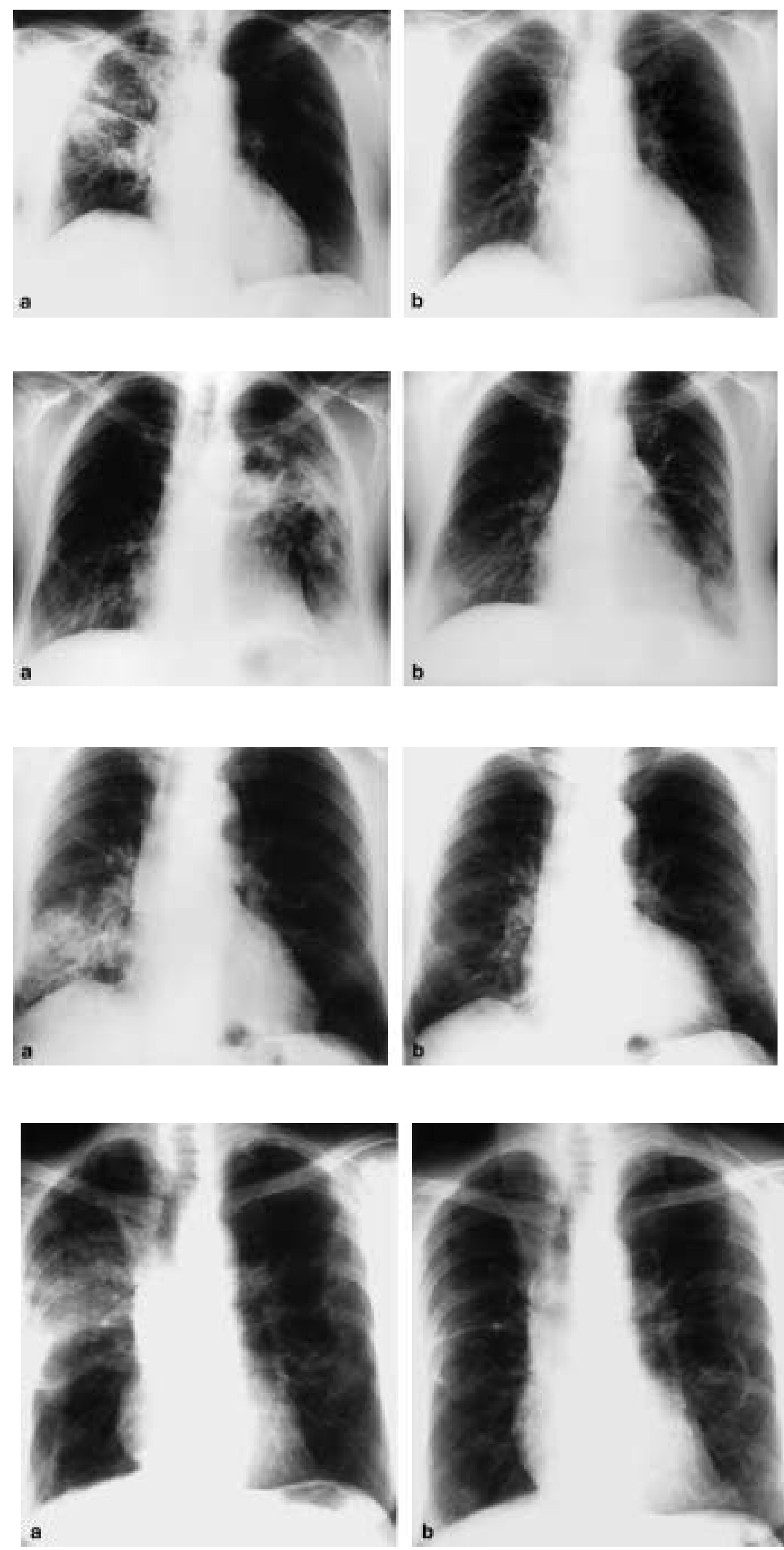

Abb. 3-6 Röntgenbilder des Thorax bei 4 Patienten mit CEP a vor und $\mathbf{b}$ nach Therapie.

Abb. 4

Abb. 5

Abb. 6

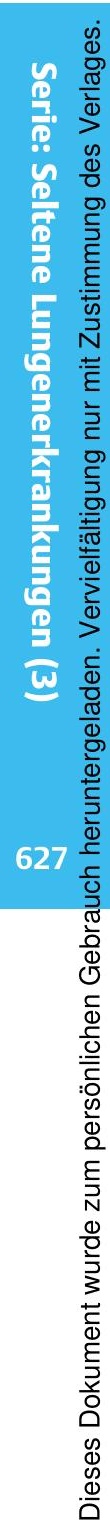




\section{Diagnostik}

Klinisch berichten die Patienten über Husten, Dyspnoe, teilweise hohes Fieber und Thoraxschmerzen, der akuten Erkrankung zwei bis drei Tage vorausgehend. Gelegentlich kommen Gelenkbeschwerden und Muskelschmerzen vor [64]. Die radiologischen Bilder zeigen bilaterale Infiltrate, alveoläre oder interstitielle Dichteanhebungen bzw. auch beides [6,16,17,27,34,44,58,64]. Selten werden auch einseitige bzw. beidseitige schmale Pleuraergüsse diagnostiziert [64]. Im Thorax-CT dominieren Groundglass-Phänomene und parenchymale Läsionen, teilweise mit Konsolidierung und kleinen Knötchen. Bei einigen Patienten kommt es zur Verdickung der Interlobulärsepten.

Die Laborwerte zeigen in der Regel eine ausgeprägte neutrophile Leukozytose. Der Gesamt-IgE-Spiegel ist bei einigen Patienten erhöht [58]. Auch das Pleuraexsudat zeigt einen hohen Eosinophilenanteil. Die Bluteosinophilie fehlt anfangs meist, später können höhere Werte im Verlauf dokumentiert werden. Die Blutgasanalyse zeigt dem schweren klinischen Krankheitsbild entsprechend eine schwere Hypoxämie, die in aller Regel eine Sauerstofftherapie, meistens auch eine Beatmungstherapie, notwendig macht. Die Lungenfunktion, wenn sie gemessen werden kann, zeigt eine restriktive Störung, die Diffusionskapazität ist deutlich erniedrigt.

Die diagnostische Methode der Wahl ist die bronchoalveoläre Lavage. Sie zeigt eine hohe Eosinophilie im Mittel von 40\% $[17,64]$. Gelegentlich finden sich zusätzlich leichte Erhöhungen der Neutrophilen und auch der Lymphozyten. Falls durchgeführt, kann die transbronchiale Biopsie die Infiltration von Eosinophilen im Interstitium und in der Bronchialwandung aufdecken [73].

\section{Verlauf und Therapie}

Trotz des schweren Krankheitsbildes kommt es unter einer Behandlung mit $1 \mathrm{mg}$ Kortison pro Kilogramm Körpergewicht zunächst alle sechs, später alle zwölf Stunden zu einer raschen initialen Besserung. Die Therapie wird dann mit deutlich niedrigeren Steroiddosen über die weiteren zwei bis vier Wochen fortgeführt. Bei dieser seltenen Erkrankung wird man keine grundsätzlichen Therapieempfehlungen aussprechen können. Die Dosis und Dauer der Steroidtherapie muss von dem aktuellen Krankheitsbild abhängig gemacht werden.

Die klinischen Unterschiede zur chronischen eosinophilen Pneumonie zeigt die Tab. 9.

Dabei sind besonders die fehlende Bluteosinophilie und das schwere Krankheitsbild gegenüber der chronischen eosinophilen Pneumonie $\mathrm{zu}$ beachten.

Im Gegensatz zur chronisch eosinophilen Pneumonie sind bisher keine Rezidive bekannt geworden.

Zusammengefasst kann man folgende diagnostische Kriterien zur Diagnose der akuten eosinophilen Pneumonie aufstellen (Tab. 10).
Tab. 9 Unterschiede zwischen akuter und chronischer eosinophiler Pneumonie

\begin{tabular}{lll}
\hline & AEP & CEP \\
\hline Geschlecht & Männer & Frauen \\
\hline Beginn & akut $(4-7 \mathrm{~d})$ & schleichend \\
\hline Bluteosinophilie & fehlt meist & in $\sim 70 \%$ vorhanden \\
schwere Hypoxämie & immer & selten \\
\hline Rezidive & keine & häufig \\
\hline
\end{tabular}

Tab. 10 Diagnostische Kriterien einer AEP (nach $[2,6,14]$ )

- akuter Beginn ohne wesentliche Vorboten

mit Husten, ausgeprägter Dyspnoe

- Fieber bis $40^{\circ} \mathrm{C}$

- bilaterale Infiltrate

- schwere Hypoxämie

- Eosinophilie in der BAL über 25\% bzw. Prädominanz der Eosinophilen im Gewebe bei offener Lungenbiopsie

- kein Hinweis auf eine Hypersensitivitäts-Reaktion

Eigene Erfahrungen bei akuter eosinophiler Pneumonie (AEP)

Im Frühjahr 1998 wurde uns aus einem auswärtigen Krankenhaus ein nahezu moribunder 30-jähriger Mann verlegt. Die Symptomatik war ganz akut 4 Tage vor der Klinikaufnahme aufgetreten. Eine Atopie mit allergischer Rhinopathie war vorbekannt. Mehrfache kombinierte antibiotische Behandlungen hatten weder das klinische Bild mit hohen, teils septischen Temperaturen beeinflussen noch die beidseitigen Infiltrationen der Lunge verändern können (s. Abb. 7). Im Gegenteil, ein Computertomogramm der Thoraxorgane zeigte ausgeprägte beidseitige Veränderungen (Abb. 8).

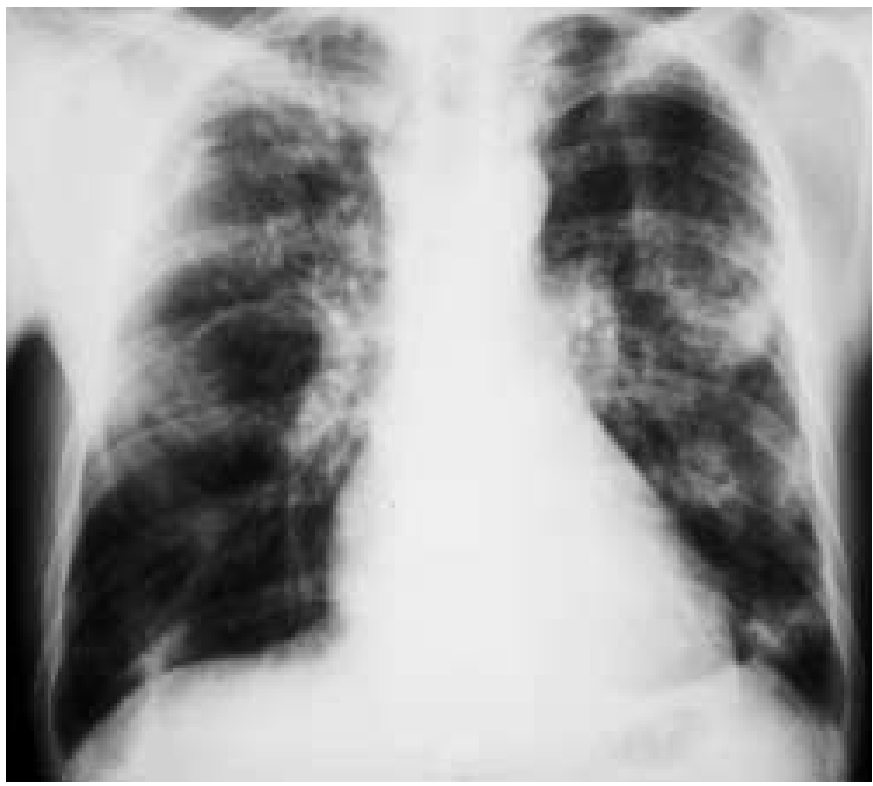

Abb. 7 Röntgen-Thoraxbild bei akuter eosinophiler Pneumonie. 


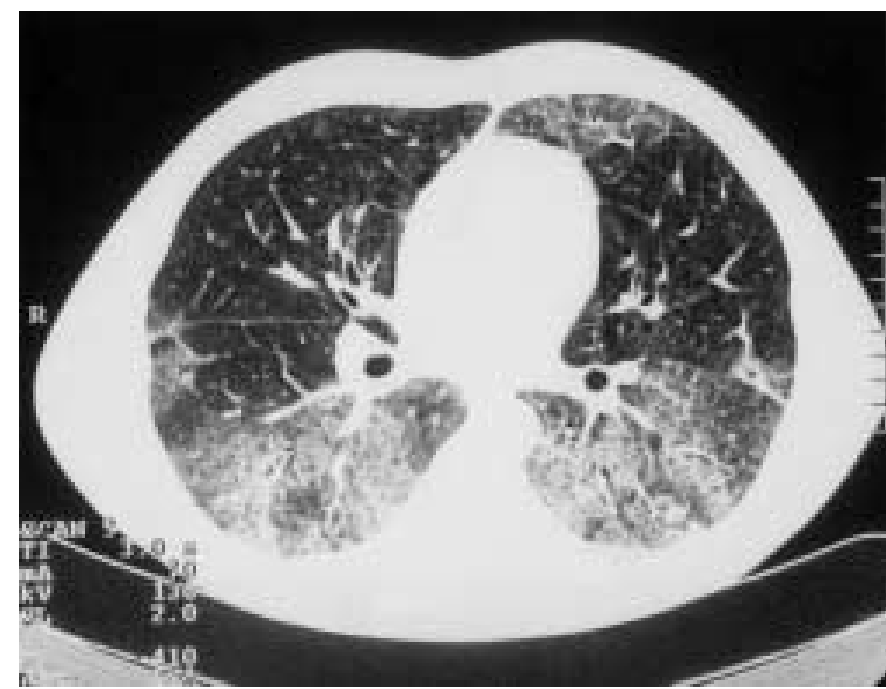

Abb. 8 CT-Thorax bei akuter eosinophiler Pneumonie.

Tab. 11 Aufnahme- und Entlassungsdaten des Patienten mit akuter eosinophiler Pneumonie

Patient N. T., 34 Jahre, bekanntes Asthma Aufnahme Entlassung (nach 10 Tagen)

\begin{tabular}{|c|c|c|}
\hline Temperatur & $38,5^{\circ} \mathrm{C}$ & $36,4^{\circ} \mathrm{C}$ \\
\hline$B S G(m m)$ & 33 & 22 \\
\hline Leukozyten & 17,9 & 8,4 \\
\hline Eosinophilie i.S. & $34 \%$ & $35 \%$ \\
\hline Röntgen-Thorax & beidseits Infiltrate & Rückbildung \\
\hline VC \% pred. & 67,8 & 95,5 \\
\hline $\mathrm{FEV}_{1} \%$ pred. & 64,4 & 102,0 \\
\hline $\mathrm{PaO}_{2}(\mathrm{~mm} \mathrm{Hg})$ & 52,1 & 88,4 \\
\hline $\mathrm{PaCO}_{2}(\mathrm{~mm} \mathrm{Hg})$ & 35,2 & 36,8 \\
\hline BAL-Zytologie & $93 \%$ Eos & - \\
\hline
\end{tabular}

Die Verlegung erfolgte zur offenen Lungenbiopsie, die dem Patienten kaum zugemutet werden konnte und auch nicht durchgeführt wurde. Die Funktionsdaten und die Ergebnisse der sofort durchgeführten BAL zeigt die Tab.11.

Diese Ergebnisse ließen keinen Zweifel an der Diagnose einer AEP, die bis dato in Mitteleuropa nicht diagnostiziert worden war.

Die am Aufnahmetag nach dem Ergebnis der BAL begonnene Steroidtherapie mit $100 \mathrm{mg}$ Kortison zeigte bereits nach 48 Stunden parallel mit der klinischen Besserung eine signifikante Anhebung des anfangs stark erniedrigten $\mathrm{PO}_{2}$. Innerhalb von einer Woche normalisierten sich alle Funktionswerte und auch das Röntgenbild fast völlig.

Ein Churg-Strauss-Syndrom schied durch den dramatischen Verlauf und das Fehlen einer systemischen Vaskulitis aus. Im Nachhinein blieben auch die Serumwerte der ANCA-Untersuchungen negativ.

\section{Literatur}

${ }^{1}$ Albera C, Ghio P, Solidoro P et al. Activated and memory alveolar T-lymphocytes in idiopathic eosinophilic pneumonia. Eur Respir J 1995; 8: 1281 - 1285

${ }^{2}$ Allen JN, Pacht ER, Gadek JE et al. Acute eosinophilic pneumonia as a reversible cause of non infectious respiratory failure. $\mathrm{N}$ Engl J Med 1989; 321: 569-574

${ }^{3}$ Aoshima M, Doi M, Usui Y et al. Allergic granulomatous angiitis associated with cerebral infarction, myo-pericarditis and acute respiratory failure due to eosinophilic pneumonia. Nihon Kokyuki Gakkai Zasshi 1998; 36: 978-983

${ }^{4}$ Azar R, Brillet L, Poret E et al. Pneumonie chronique à éosinophiles. Semin Hop Paris 1990; 66: 2491 -2494

${ }^{5}$ Bain GA, Flower CD. Pulmonary eosinophilia. Eur J Radiol 1996; 23 : 3-8

${ }^{6}$ Balbi B, Fabiano F. A young man with fever, dyspnoea and nonproductive cough. Eur Respir J 1996; 9: 619-621

${ }^{7}$ Bancal C, Sadoun D, Valeyre D et al. Pneumopathie chronique idiopathique à éosinophiles: maladie de Carrington. Presse Med 1989; 18: $1695-1698$

${ }^{8}$ Barnes N, Gray BJ, Heaton R et al. Pulmonary eosinophilia in identical twins. Thorax 1983; 38: $318-319$

${ }^{9}$ Beninati W, Derdak S, Dixon PF et al. Pulmonary eosinophils express HLA-DR in chronic eosinophilic pneumonia. J Allergy Clin Immunol 1993; 92: $442-449$

${ }^{10}$ Bentur L, Bar-Kana Y, Lirni E et al. Severe minocycline-induced eosinophilic pneumonia: extrapulmonary manifestations and the use of in vitro immunoassays. Ann Pharmacother 1997; 31: 733 - 735

${ }^{11}$ Boomars KA, van Velzen-Blad H, Mulder PG et al. Eosiniphil cationic protein and immunoglobulin levels in bronchoalvolar lavage fluid obtained from patients with chronic eosinophilic pneumonia. Eur Respir J 1996; 9: $2488-2493$

12 Boon ES, Graal MB, van Noord JA. Primary extranodal Non-Hodgkin's lymphoma of the lung presenting with bilateral, patchy infiltrates dramatically improving after corticosteroid therapy. Chest 1993; 104: $1292-1293$

${ }^{13}$ Brezis M, Lafair J. Thrombocytosis in chronic eosinophilic pneumonia. Chest 1979; 76: $231-232$

${ }^{14}$ Buchheit J, Eid N, Rodgers G et al. Acute eosinophilic pneumonia with respiratory failure: a new syndrome? Am Rev Respir Dis 1992; 145: $716-718$

${ }^{15}$ Carrington C, Addington W, Goff A et al. Chronic eosinophilic pneumonia. N Engl J Med 1969; 280: 787 - 798

${ }^{16}$ Cheon JE, Lee KS, Jung GS et al. Acute eosinophilic pneumonia: radiographic and CT findings in six patients. AJR 1996; 167: 1195-1199

${ }^{17}$ Chiappini J, Arbib F, Heyraud JD et al. Pneumopathie éosinophilique subaiguë idiopathique d'évolution favorable sans corticothérapie. Rev Mal Respir 1995; 12: 25-28

${ }^{18}$ Cordier J-F. Eosinophilic Pneumonias. In: Schwarz MI, King jr TE (eds.). Interstitial Lung Diseases. 3rd ed. London: B. C. Dekker Inc. Hamilton, 1998: $557-595$

${ }^{19}$ Costabel W, Teschler H, Guzman J. Bronchiolitis obliterans organizing pneumonia (BOOP): the cytological and immunocytological profile of bronchoalveolar lavage. Eur Respir J 1992; 5: 791 - 797

${ }^{20}$ Crofton JW, Livingstone JL, Oswald NC et al. Pulmonary eosinophilia. Thorax 1952; 7: 1

21 Dail DH. Eosinophilic infiltrates. In: Dail DH, Hammar SP (eds). Pulmonary pathology. 2nd ed. New York: Springer-Verlag, 1994: 537-566

22 Davis WB, Wilson HE, Wall RL. Eosinophilic alveolitis in acute respiratory failure. A clinical marker for a non-infectious etiology. Chest 1986; 90: 7-10

${ }^{23}$ Dejaegher P, Demedts M. Bronchoalveolar lavage in eosinophilic pneumonia before and during corticosteroid therapy. Am Rev Respir Dis 1984; 129: $631-632$

${ }^{24}$ Demedts M, de Man F. Circulation immune complexes in chronic eosinophilic pneumonia. Acta Clin Belg 1991; 46: 75-81

${ }^{25}$ Durieu J, Wallaert B, Tonnel AB. Long-term follow-up of pulmonary function in chronic eosinophilic pneumonia. Groupe d'Etude en $\mathrm{Pa}$ thologie Interstitielle de la Société de Pathologie Thoracique du Nord. Eur Respir J 1997; 10: 286-291

${ }^{26}$ Ebara H, Ikozoe J, Johkoh T et al. Chronic eosinophilic pneumonia: evolution of chest radiograms and CT features. J Comput Assist Tomogr 1994; 18: 737-744 
${ }^{27}$ Elcadi T, Morcos E, Lancrenon C et al. Syndrome douloureux abdominal révélant une pneumonie aigue à éosinophiles. Presse Med 1997; 26: 416

${ }^{28}$ Flint AF, Colby TV. The pulmonary eosinophilias, hypersensitivity pneumonitis, and drug-induced lung diseases. In: Flint A, Colby TV (eds.). Surgical pathology of diffuse infiltrative lung disease. Orlando, Florida: Grune \& Stratton, 1987: 63-90

${ }^{29}$ Fox B, Seed W. Chronic eosinophilic pneumonia. Thorax 1980; 35 : $570-580$

${ }^{30}$ Franco J, Artes MJ. Pulmonary eosinophilia associated with montelukast. Thorax 1999; 54: $558-560$

${ }^{31}$ Gaudenz R, Hartmann K, Reinhart WH et al. Extensive eosinophilic pulmonary infiltrates in a depressive patient treated with maprotiline. Schweiz Rundsch Med Prax 1999; 88: 1047-1051

${ }^{32}$ Golstein MA, Steinfeld S. Chronic eosinophilic pneumonia followed by Churg-Strauss syndrome. Rev Rhum Engl Ed 1996; 63: 624-628

${ }^{33}$ Greenburg M, Schiffman RL, Geha DG. Acute eosinophilic pneumonia. N Engl J Med 1990; 322: 635

${ }^{34}$ Greif J, Kivity S, Struhar D et al. Bronchoalveolar lavage: a useful tool in the diagnosis of eosinophilic pneumonia. Isr J Med Sci 1986; 22: $497-480$

${ }^{35}$ Hayakawa H, Sato A, Toyoshima M et al. A clinical study of idiopathic eosinophilic pneumonia. Chest 1994; 105: 1462 - 1466

${ }^{36}$ Iwami T, Umemoto S, Ikeda K et al. A case of acute eosinophilic pneumonia. Evidence for hypersensitivity-like pulmonary reaction. Chest 1996; 110: 1618 - 1621

37 Janin A, Torpier G, Courtin P et al. Segregation of eosinophil proteins in alveolar macrophage compartments in chronic eosinophilic pneumonia. Thorax 1993; 48: 57-62

38 Jederlinic PJ, Sicilian L, Gaensler EA. Chronic eosinophilic pneumonia. A report of 19 cases and a review of the literature. Medicine 1988; 67: $154-162$

${ }^{39}$ Johkoh T, Nuller NL, Akira M et al. Eosinophilic lung diseases: diagnostic accuracy of thin-section CT in 111 patients. Radiology 2000; 216: $773-780$

${ }^{40}$ Kadokura M, Colby TV, Myers JL et al. Pathologic comparison of videoassisted thoracic surgical lung biopsy with traditional open lung biopsy. J Thorac Cardiovasc Surg 1995; 109: 494-498

${ }^{41}$ Katzenstein AL. Katzenstein and Askin's surgical pathology of nonneoplastic lung disease. $3^{\text {rd }}$ Ed. Philadelphia: WB Saunders, 1997

42 Khoo JB, Pay KH, Poh WT. Clinics in diagnostic imaging. Chronic eosinophilic pneumonia (CEP). Singapore Med J 1996; 37: 532 - 534

${ }^{43}$ Killen JW, Swift GL, White RJ. Eosinophilic fasciitis with pulmonary and pleural involvement. Postgrad Med J 2000; 76: 36-37

${ }^{44}$ King MA, Pope-Harman AL, Allen JN et al. Acute eosinophilic pneumonia: radiologic and clinical features. Radiology 1997; 203: 715 - 719

${ }^{45}$ Kirsten D, Nowak D, Rabe K et al. Die chronische eosinophile Pneumonie. Unveröffentlichte Daten,

${ }^{46} \mathrm{Kita}$ H, Sur S, Hunt LW et al. Cytokine production at the site of disease in chronic eosinophilic pneumonitis. Am J Respir Crit Care Med 1996; 153: $1437-1441$

${ }^{47}$ Krupsky M, Landau Z, Lifschitz-Mercer B et al. Wegener's granulomatosis with peripheral eosinophilia. Chest 1993; 104: 1290-1292

${ }^{48}$ Lavandier M, Carre P. Effectiveness of inhaled high-dose corticosteroid therapy in chronic eosinophilic pneumonia. Chest 1994; 105: $1913-1914$

${ }^{49}$ Liebow AA, Carrington CB. The eosinophilic pneumonias. Medicine 1969; 48: $251-285$

${ }^{50}$ Marchand E, Reynaud-Gaubert M, Lauque D et al. Idiopathic chronic eosinophilic pneumonia (CEP). A clinical and follow-up study of 62 cases. Medicine 1998; 77: 299-312

${ }^{51}$ Matsuyama W, Mizoguchi A, Iwami F et al. A case of pulmonary infiltration with eosinophilia caused by Ascaris suum. Nihon Kokyuki Gakkai Zasshi 1998; 36: 208 - 212

52 Mayo J, Müller N, Road JE. Chronic eosinophilic pneumonia. CT findings in six cases. AJR 1989; 153: 727-730

${ }^{53}$ McCarthy DS, Pepys J. Cryptogenic pulmonary eosinophilias. Clin Allergy 1973; 3: 339-351

${ }^{54}$ Meyers JL, Katzenstein AL. Granulomatous infection mimicking bronchocentric granulomatosis. Am J Surg Pathol 1986; 10: 317 - 322
${ }^{55}$ Mukae H, Kadota JI, Kohno S et al. Increase of activated T-cells in BAL fluid of Japanese patients with bronchiolitis obliterans organizing pneumonia and chronic eosinophilic pneumonia. Chest 1995; 108: $123-128$

${ }^{56}$ Naughton M, Fahy J, Fitzgerald MX. Chronic eosinophilic pneumonia. A long-term follow-up of 12 patients. Chest 1993; 103: 162-165

${ }^{57}$ Nishimura K, Izumi T, Kitaichi M et al. The diagnostic accuracy of highresolution computed tomography in diffuse infiltrative lung disease. Chest 1993; 104: 1149-1155

${ }^{58}$ Ogawa H, Fujimura M, Matsuda T et al. Transient wheeze. Eosinophilic bronchobronchiolitis in acute eosinophilic pneumonia. Chest 1993; 104: $493-496$

59 Orliaguet O, Langin T, Gavazzi G et al. Acute eosinophilic pneumonia due to hypersensitivity to carbamazepine. Rev Mal Respir 1998; 15: $797-799$

${ }^{60}$ Oshikawa K, Kuroiwa K, Tokunaga T et al. Acute eosinophilic pneumonia with increased soluble ST2 in serum and bronchoalveolar lavage fluid. Respiratory Medicine 2001; 95: 532 -533

${ }^{61}$ Pacheco A, Cuevas M, Carbelo B et al. Eosinophilic lung disease associated with Candida albicans allergy. Eur Respir J 1998; 12: 502 - 504

${ }^{62}$ Pearson DJ, Rosenow EC. Chronic eosinophilic pneumonia (Carrington's). A follow-up study. Mayo Clin Proc 1978; 53: $73-78$

${ }^{63}$ Pesci A, Bertorelli G, Manganelli P et al. Bronchoalveolar lavage in chronic eosinophilic pneumonia. Analysis of six cases in comparison with other interstitial lung diseases. Respiration 1988; 54 (Suppl. 1): $16-22$

${ }^{64}$ Pope-Harman AL, Davis WB, Allen ED et al. Acute eosinophilic pneumonia. A summary of 15 cases and review of the literature. Medicine 1996; 75: 334-342

65 Potter MB, Fincher RK, Finger DR. Eosinophilia in Wegener's granulomatosis. Chest 1999; 116: 1480-1483

${ }^{66}$ Reeder WH, Goodrich BE. Pulmonary infiltration with eosinophilia (PIE syndrome). Ann Intern Med 1952; 36: 1217-1240

${ }^{67}$ Shijubo N, Fujishima T, Morita S et al. Idiopathic chronic eosinophilic pneumonia associated with noncaseating epithelioid granulomas. Eur Respir J 1995; 8: 327 - 330

68 Speich R, Hess T, Krestin GP et al. The value of bronchoalveolar lavage for the diagnosis of eosinophilic pneumonias. Schweiz Med Wochenschr 1992; 122: $1005-1010$

${ }^{69}$ Steinfeld S, Golstein M, de Vuyst P et al. Chronic eosinophilic pneumonia (CEP) as a presenting feature of Churg-Strauss syndrome (CSS). Eur Respir J 1994; 7: 2098

70 Suganuma H, Inui N, Sato J et al. A case of eosinophilic pneumonia associated with Mycobacterium avium infection. Kekkaku 1995; 70 : $355-360$

${ }^{71}$ Tani K, Kashio M, Sano N et al. A case of sarcoidosis associated with chronic eosinophilic pneumonia. J Med Invest 1998; 45: 131 - 136

72 Tanigawa K, Sugiyama K, Matsuyama $\mathrm{H}$ et al. Mesalazine-induced eosinophilic pneumonia. Respiration 1999; 66: 69-72

73 Tazelaar HD, Linz LJ, Colby TV et al. Acute eosinophilic pneumonia: histopathologic findings in nine patients. Am J Respir Crit Care Med 1997; 155: 296-302

74 Watanabe K, Nishimura K, Shiode M et al. Captopril, an angiotensinconverting enzyme inhibitor, induced pulmonary infiltration with eosinophilia. Intern Med 1996; 35: $142-145$

${ }^{75}$ Yoshida K, Shijubo N, Koba H et al. Chronic eosinophilic pneumonia progressing to lung fibrosis. Eur Respir J 1994; 7: 1541 - 1544

Bereits publizierte Beiträge zu dieser Serie:

1. Pulmonale Lymphangioleiomyomatose. Pneumologie 2002; 56: $309-315$

2. Alveolarproteinose. Pneumologie. 2002; 56: 448 - 456 